\title{
Biochemical and Metabolical Pathways Associated with Microbiota-Derived Butyrate in Colorectal Cancer and Omega-3 Fatty Acids Implications: A Narrative Review
}

\author{
Adelina Silvana Dragomir ${ }^{1,+}$, Șerban Mircea Negru 2,*,t, Mădălina Preda ${ }^{3, *}$, Raluca Ioana Mihăilă ${ }^{1}$, Isabela Anda \\ Komporaly 1, Elena Adriana Dumitrescu 1, Cristian Virgil Lungulescu 4, Lidia Anca Kajanto 1, Bogdan Georgescu 1, \\ Emanuel Alin Radu ${ }^{1}$, Dana Lucia Stănculeanu ${ }^{1}$
}

\begin{abstract}
1 Department of Oncology, "Carol Davila" University of Medicine and Pharmacy, 020021 Bucharest, Romania; adelina-silvana.gheorghe@drd.umfcd.ro (A.S.D.); raluca-ioana.mihaila@drd.umfcd.ro (R.I.M); isabelaanda.komporaly@drd.umfcd.ro (I.A.K.); elena-adriana.dumitrescu@drd.umfcd.ro (E.A.D.); lidia.kajanto@drd.umfcd.ro (L.A.K.); bogdan.georgescu@drd.umfcd.ro (B.G.); alin.radu@umfcd.ro (E.A.R.); dana.stanculeanu@umfcd.ro (D.L.S.)

2 Department of Oncology, "Victor Babeș" University of Medicine and Pharmacy, 300041 Timisoara, Romania; serban.negru@oncohelp.ro

3 Department of Microbiology, Parasitology and Virology, Faculty of Midwives and Nursing, Carol Davila University of Medicine and Pharmacy, 020021 Bucharest, Romania; madalina.preda@drd.umfcd.ro

4 Department of Medical Oncology, University of Medicine and Pharmacy Craiova, 200349 Craiova, Romania; cristilungulescu@yahoo.com

* Correspondence: serban.negru@oncohelp.ro, madalina.preda@drd.umfcd.ro

+ These authors contributed equally to this work.
\end{abstract}

\begin{abstract}
Knowledge regarding the influence of the microbial community in cancer promotion or protection has expanded even more through the study of bacterial metabolic products and how they can modulate cancer risk, which represents an extremely challenging approach for the relationship between intestinal microbiota and colorectal cancer (CRC). This review discusses research progresses in the effect of bacterial dysbiosis from a metabolic point of view, particularly on the biochemical mechanisms of butyrate, one of the main short chain fatty acids (SCFAs) with anti-inflammatory and anti-tumor properties in CRC. Increased daily intake of omega-3 polyunsaturated fatty acids (PUFAs) significantly increases the density of bacteria that are known to produce butyrate. Omega-3 PUFAs have been proposed as a treatment to prevent gut microbiota dysregulation and lower the risk or progression of CRC.
\end{abstract}

Keywords: Butyrate; Colorectal cancer; Gut microbiota; Diet; omega-3 PUFAs

\section{Introduction}

According to the estimates of cancer incidence and mortality produced by the International Agency for Research on Cancer (GLOBOCAN) for 2020, colorectal cancer (CRC) represents one of the major health threats, especially in developed countries which are at higher risk, due to the dietary and lifestyle patterns of the population. [1,2] It is the second leading cause of cancer death worldwide, and the third most diagnosed cancer, while having a remarkable geographical variation. [2,3] As the global burden of CRC is expected to increase by $60 \%$ until 2030, with incidence and mortality rates rapidly rising in many countries, in direct correlation with economic development and environmental changes, targeted interventions are necessary in order to reduce the number of patients in the future years. [4]

Complex genetic and epigenetic alterations contribute to the heterogeneity of CRC, having a multifactorial etiology and a wide variety of risk factors. [3,5] Besides the chromosomal and molecular characteristics involved in tumors arising from colon and rectum, which have genetic signatures belonging to three major pathways (chromosomal instability, mismatch repair and $\mathrm{CpG}$ island methylator phenotype), outstanding evidence-based on global scientific and epidemiological studies emphasizes on the role of environmental 
or lifestyle modifiable risk factors, such as sedentary lifestyle or physical inactivity, obesity, smoking, processed food, alcohol and meat consumption. [3,5] The human microbiome has a well-established impact on human physiology and disease, emerging in the last years as one of the factors involved in oncogenesis. There is strong evidence that indicates how gut microbiota can influence the development of CRC, through multi-step disturbances of composition or function. [6] Recent advances and accumulating evidence of pathogens' pro-carcinogenic effects, by promoting chronic inflammation, DNA alteration and activation of anti-apoptotic signaling pathways in malignant cells have contributed to understanding the etiology of several types of cancer, including CRC. $[7,8]$ Pathogenic bacteria contribute to the pro-tumorigenic microenvironment through different mechanisms, such as outcompeting commensals, altering $\mathrm{pH}$, secreting toxins or possessing virulence factors which can disrupt the epithelial barrier and activate the inflammation cascade. [7]

The first consensus led by a group of 18 experts on the cancer-associated microbiome had been published in 2019. More than half (10) agreed that there is a causal relationship between the human microbiome and the etiopathogenesis of some cancers. [9] The consensus acknowledged the lacks in the existing evidence and the need for future research in the field. The conclusions were that the microbiome, the environmental factors and an epigenetically or genetically vulnerable host, represents one of the three parts of the multidirectional "interactome" / "interome" leading to carcinogenesis. [9]

\section{The gut microbiome and colorectal cancer}

The $20^{\text {th }}$ century has brought an incomparable revolution in understanding the microbiota (formed by commensal symbionts, pathogens or pathobionts) and its associations to more than 100 essentially different disorders. [10] A comprehensive review, in which each microorganism was associated with various diseases, local or external factors and antibiotic consumption, has recently outlined the global picture of the human microbiota composition (community of microbes residing in and on the human body) and function of the microbiome, defined as the combined genetic material of the microbiota. [11]

There was a long process that led to current insights, as the first descriptions of the human microbiota date back to the 1680s, when Antonie van Leeuwenhoek began to use the microscope invented by himself to describe and illustrate his own bacteria in the swabs from his oral mucosa and faeces. [10] Almost 200 years later, the book published by Joseph Leidy can be considered the "birth" of microbiota research. It was followed by the work of Pasteur, Metchnikoff, Koch, Escherich, Kendall and many others, who laid the groundwork for the present understanding of microorganism-host interactions. [10] The third millennium can be considered so far "the golden age" of microbiota research, as it brought extensive population-based studies, such as the Human Microbiome Project (started five years after the completion of the Human Genome Project) or the American Gut Project.

Millions of years of co-evolution between host and microorganisms led to the formation of a mutualistic relationship, a complex interplay between the host immune system and the microbiota, essential for gut homeostasis. [12] In this interaction, microbiota's main contributions to the host are the digestion and fermentation of carbohydrates, production of vitamins, development of gut-associated lymphoid tissues, the polarization of gut-specific immune responses and prevention of colonization by pathogens. In contrast, the gut immune responses induced by commensal bacteria can regulate the composition of the microbiota. [13] There is also a bidirectional relationship between bacteria and metabolites, with bacteria influencing metabolite composition of the gut and metabolites contributing to the architecture of microbiota. [14]

Molecular approaches through metagenomics (sequencing of all DNA present in a sample) have firstly characterized the genetic background (gene composition) of the microbiome, offering an in-depth understanding of the complex and diverse bacterial communities. [15] However, the need for additional analysis of other datasets has rapidly emerged, as more knowledge was required about the host-microorganisms interplay, because metagenomic assays could not capture some bacterial features, they could not detect 
minority populations and could not discriminate between live bacteria and transient DNA. [12] Therefore, researchers have used broad "meta-omics" technologies integrated with metagenomic data, such as metatranscriptomics (sequencing of all mRNA generated to determine gene expression), culturomics (culturing approach using multiple culture conditions, MALDI-TOF mass spectrometry and 16S rRNA sequencing), metaproteomics (analysis of microbial proteins that are actively synthesised by the microbiota), toxicogenomics (DNA-DNA hybridization, DNA G + C content and 16S rRNA sequence similarity) and metabolomics (the study of all chemical processes concerning microbiota's metabolites). [11,16,17]

Studying the metabolic pathway information of the gut microbiota is particularly unique, as metabolites are considered to be universal, while proteins and genes vary across taxa. More research should be focused on gaining knowledge of the important mutual dependence between mucosal gut-associated metabolome composition and gut microbiome, manifested by two general processes: the first one represented by catabolism and anabolism of metabolites by microbes and the second by stimulation and inhibition of microbial growth by metabolites. [18] This interomic integrative analysis is required in order to transform the metabolites into a specific target for diagnosing or monitoring CRC or other microbiome-associated intestinal diseases and provide a potential therapeutic intervention (either directly or indirectly, through diet). [18]

To understand microbiota's metabolic transformation capabilities and how they can affect the host is essential to study the knowledge gap in the molecular basis for gut microbiota-nutrient interactions (nutritional requirements, links between diet and disease, the effect of diet on microbiota) also from a biochemical point of view, in strong correlation with host nutrition. [19] The altered microbiota can lead to a disruption in the mucosal barrier, promote or inhibit tumorigenesis through different immune responses and microbiome-derived metabolites. [20] On the one hand, there are some microbial metabolites, such as prostaglandin E2 and secondary bile acids associated with increased risk of CRC and on the other one, some are associated with decreased CRC risk: indole, anti-oxidants, short-chain fatty acid (SCFAs) and ursodeoxycholic acid. [21,22]

A chronic state of unhealthy and dysbiotic acquired microbiota, defined as aberrant composition and function, with a high resilience potential, has been shown to contribute to cancer pathogenesis, both directly and indirectly via: prolonged inflammation, promotion of cell growth and proliferation, changes in immune responses (lessening the strength of immunosurveillance), metabolic changes (alteration of food and drug metabolism or other biochemical functions of the host), DNA damage and alterations of the anti-cancer therapy efficacy. $[13,23]$ Microbial dysbiosis has intricate connections with neoplastic diseases, especially with CRC, as the most important and developed community of human microbiota resides in the gastrointestinal tract.

However, recent research has also identified several mechanisms through which gut microbiota may support the host's fight against cancer, such as the use of antigenic mimicry, biotransformation of chemotherapeutic agents, boosting of anti-cancer immune responses (to improve the efficacy of cancer immunotherapy) and producing microbial metabolites with tumor suppressing properties. [23]

Additionally, to the laboratory research, there are many investigations currently taking place also in the clinical setting. Prospective cohort studies of patients with CRC could distinguish early-stage patients from more advanced disease, based on the gut microbiome and metabolome. For example, a clinical trial (NCT04005742) with unpublished results yet, aims to perform additional measurements to the ones in the BORICC study (Biomarkers Of Risk of Colorectal Cancer), including the gut microbiome, faecal SCFA concentrations and expression of genes associated with CRC, in a 12+ year follow-up longitudinal study (BFU - BORICC Follow-Up). [24] The ultimate goal of BFU study is to identify lifestyle factors able reduce CRC risk while characterizing the underlying mechanisms in which lifestyle and ageing affect CRC risk, in order to design better early prevention strategies. [24] 


\section{Microbiota-derived butyrate in colorectal cancer}

SCFAs are weak organic acids with between 2 and 5 carbon molecules, including acetate $(\mathrm{C} 2)$, propionate $(\mathrm{C} 3)$, butyrate $(\mathrm{C} 4)$, and valerate $(\mathrm{C} 5)$, which are produced by intestinal bacterial fermentation of mainly undigested dietary carbohydrates (especially resistant starches and dietary fibers). [25] However, they also result in small quantities from dietary and endogenous proteins, through a pathway that also produces toxic nitrogenous and sulfur metabolites, such as ammonia (from the conversion of the amino acid lysine into butyrate). [26] Concentration ratio in the colonic lumen of the three main SCFAs is about $3 / 1 / 1: 60 \%$ acetate, $25 \%$ propionate and $15 \%$ butyrate, the last one being the preferred energy source utilized by epithelial cells from the colon and only small proportions reach the portal vein or the systemic circulation. [25]

In vitro studies have demonstrated the important role of butyric acid in the prevention of CRC. An evaluation of HCT116 human CRC cells treated with butyric acid derivatives proved that apoptosis is induced in the cancer cells by activation of caspase- 3 activity and induced cell cycle arrest. [27] Moreover, SCFAs can also regulate the expression of inflammatory cytokines and chemokines by the colonic epithelial cells in different immune processes, having a role in gut homeostasis and promoting the integrity of the intestinal barrier. [28] The effect of butyrate on the epithelial integrity has been observed also on animal models, where it contributes to the healing of colonic tissue at the anastomosis sites after surgery for CRC. [29]

Butyrate-producing bacteria are an abundant and phylogenetically diverse group of microorganisms, considered rather a functional group of Gram-positive anaerobic Firmicutes, which play an important role in maintaining a healthy gut, primarily through their production of butyrate. $[30,25]$ Two of the most numerically important groups are considered to be Faecalibacterium prausnitzii, belonging to the Clostridium leptum cluster (clostridial cluster IV) and Eubacterium rectale/Roseburia spp., belonging to the Clostridium coccoides cluster (clostridial cluster XIVa). [30] There are two microbial enzymes responsible for the final synthesis of butyrate from two molecules of acetyl-CoA: butyryl-CoA transferase (dominant, formed by a variety of genera and species) and butyrate kinase (favored in proteolytic fermentation). [6]

Increased butyrate production has often been hypothesized to be one of the beneficial effects of prebiotics and probiotics. [28] In a clinical trial (NCT03072641) which aimed to determine whether probiotic bacteria have a beneficial effect on the CRC-associated microbiota, researchers used dietary supplementation with Bifidobacterium lactis Bl-04 and Lactobacillus acidophilus NCFM and analyzed the microbiota composition in tissue and faeces samples, at baseline and after probiotics use. [31] The results showed that patients with CRC who received probiotics had an increased abundance of butyrate-producing bacteria (especially Faecalibacterium spp. and Clostridiales spp.) in the samples from the tumor, nontumor mucosa and faeces, compared with the group that did not receive probiotics. [31] Moreover, CRC-associated taxa (Fusobacterium and Peptostreptococcus) were less frequent in the faecal samples of patients who received probiotics, upholding the hypothesis that CRC-associated microbiota can be manipulated by specific probiotic strains and providing hope that probiotics modulation of microbiota could be considered an integrative part of the therapeutic approach for CRC patients. [31]

The structure of microbiota in patients with CRC was described as significantly different from the one encountered in healthy individuals. There are several studies of butyryl-CoA: acetate CoA-transferase gene quantification from the gut microbial population and they all found that butyrate-producing bacteria (such as Ruminococcus spp. and Pseudobutyrivibrio ruminis) are reduced in the faeces of CRC patients, pointing out the benefits of bacterial metabolites. [21,32,33] An evaluation of diet and age suggested that these factors also influence the level of butyrate-producing bacteria in the gut - the older participants had significantly fewer copies of the butyryl-CoA: acetate CoA-transferase gene than young omnivores, while vegetarians showed the highest number. [34] This fact may 
reflect the increased risk for CRC in the elderly, due to their low butyrate production capacity and the protective effect of a vegetarian diet against CRC.

In a study that aimed to describe how microbial functions may influence CRC development, researchers used stool profiling to identify intestinal microbiome and metabolome and analyze the different representation in humans with CRC, compared to healthy controls. [21] They quantified several SCFAs from frozen stool samples, among which, acetic and valeric acids were significantly higher in the faeces from CRC patients. In contrast, butyric acid was significantly higher in the healthy samples, and propionic acid was detected in similar quantities between the two groups. [21] Acetate can be turned into butyrate, but the proportional differences in these two SCFAs metabolites between CRC and healthy individuals may be explained by a reduction of gut bacteria that can perform this reaction in CRC samples. Otherwise, it may be a result of the conversion of butyrate into acetate, a degradation process that takes place under the acidic (low) colonic $\mathrm{pH}$ induced by the tumor. [21] In CRC samples, significantly higher relative concentrations of isobutyric and isovaleric acid were observed as well, being products from the bacterial metabolism of branched-chain amino acids valine and leucine, also higher in CRC stool samples. [21]

Butyrate proved to be more than just a metabolite, having as well important cellular signaling roles, linked to epigenetic regulations of gene expression. There are two main forms of epigenetic changes encountered in CRC (and many other cancers), defined as chemical alterations to DNA or chromatin that do not affect the primary DNA sequence: those that directly modify DNA (DNA hypo- or hypermethylation) and those that modify DNA-binding proteins (histone modifications - methylation or demethylation and the acetylation or deacetylation). [35] These changes alter the regulation and expression of genes and other DNA elements in a predictable fashion and are reversible, unlike changes to the genomic sequence. $[36,35]$ The enzymes that catalyze histone acetylation are called histone acetyltransferases (HATs) and the ones that catalyze the removal of an acetyl group from a histone are called histone deacetylases (HDACs), both playing a crucial role in the remodeling of chromatin. [36] Their enzymatic activities induce structural alterations of histones, enabling access of transcription factors to a portion of DNA chromatin, influencing the transcription and expression of a given gene. [36]

Butyrate was the first identified endogenous HDACI, back in the year 1977 and for more than two decades, it was the only one available for research, with the primary target in the clinical development of cancer treatment. [37] During the last decade, inhibition of HDACs by HDAC inhibitors (HDACIs) emerged as a target for specific epigenetic modification associated with cancer or other diseases (hemoglobinopathies, cystic fibrosis, Xlinked adrenoleukodystrophy, muscular dystrophies, neurodegenerative disorders, systemic lupus erythematosus etc.). More than 20 substances have entered clinical studies by now, while some have already been approved (for example vorinostat/suberanilohydroxamic acid and romidepsin/depsipeptide for the treatment of cutaneous T-cell lymphoma or panobinostat for the treatment of multiple myeloma). [38]

Due to the fact that HDACs are key enzymes for regulating cell death and have a role in promoting carcinogenesis, HDACIs have been exploited for their role in cancer therapy and have been shown to regulate the survival of tumor-infiltrating T lymphocytes (TILs), by suppressing their apoptosis. [39] HDACIs, particularly butyrate, also inhibit directly colon carcinogenesis, by decreasing the expression of cyclin B1 gene (a cell cycle promoter) in colon cancers cells, as shown in vitro. [40] Moreover, co-administration of HDACIs and anti-CTLA4 (cytotoxic T-lymphocyte antigen 4 ) antibodies seems to act synergistically for the therapeutic effect, enhancing T-cell infiltration within tumor and the anti-tumor immune response. [39] Another mechanism by which microbiota-derived butyrate promotes cellular metabolism is the enhancement of memory potential in activated CD8 ${ }^{+} \mathrm{T}$ cells (through increased oxidative and glycolytic activity, improved mitochondrial mass and membrane potential), with implications in immunotherapy and vaccination. [41]

Belcheva et al. conducted a study on the murine model that investigated the ability of gut microbiota to synergize with mutations in oncogenes or tumor suppressor genes 
(APC, MSH), as well as with established lifestyle risk factors related to diet (high-carbohydrate intake formed by starch and sucrose), has demonstrated the role of microbialderived butyrate in the straightforward connection between host genetics and gut microbes. [42] Some of the most common genetic changes involved in this pathology are the mutation in or silencing of the genes involved in DNA mismatch repairs (MMR) mechanisms, such as MutS homolog 2 (MSH2) and the mutation of adenomatous polyposis coli (APC) tumor suppressor gene, which regulates $\mathrm{Wnt} / \beta$-catenin pathway. [43] There is important research concern over carbohydrate-rich diets; for example, a meta-analysis of 39 studies suggested an overall direct association between glucose metabolism factors (glycemic index, glycemic load) and CRC risk. [44] In a prospective, observational study of participants from the National Cancer Institute-sponsored Cancer and Leukemia Group B (CALGB) 89803 trial (ClinicalTrials.gov identifier NCT00003835), regarding patients with stage III colon cancer, it had been stated that total carbohydrate intake and increased dietary glycemic load were significant statistically associated with a higher risk of recurrence and mortality. [45]

The mentioned study of Belcheva et al. was conducted on APC ${ }^{\mathrm{Min} /+}$ (multiple intestinal neoplasia) mouse, a well-established animal model of human adenomatous polyposis, with MSH2 deficiency: an APC ${ }^{\mathrm{Min} /+} \mathrm{MSH}^{-/-}$mouse model of CRC. [42] Alteration of the gut bacterial community structure with antibiotics led to decreased polyp numbers in the mice colons, without a reduction in the abundance of colonic bacteria, through a mechanism independent of both inflammation and DNA damage. [42] Putting mice on 7\% lowcarbohydrate diet led to substantial changes in the relative proportions of the bacterial phyla but did not alter the total bacterial abundance. Moreover, this diet reduced the number of polyps in the digestive tracts of $\mathrm{APC}^{\mathrm{Min} /+} \mathrm{MSH}^{-/-}$mice, in a similar amount achieved by treating mice with antibiotics. [42] The antibiotic treatment and the low-carbohydrate diet had also numerous other effects observed in $\mathrm{APC}^{\mathrm{Min} /+} \mathrm{MSH} 2^{-/-}$mice: both interventions reduced the number of cells with DNA breaks, reduced Ki-67 expression, modulated and restored the nuclear $\beta$-catenin expression to that encountered in $\mathrm{APC}^{\mathrm{Min} /+} \mathrm{MSH} 2^{+-}$ mice, reduced the production of numerous metabolites (lactate, only butyrate from all SCFAs, uracil, xanthine etc.) of microbial fermentation, decreased three butyrate-producing families within Firmicutes phylum (Clostridiaceae, Lachnospiraceae and Ruminococcaceae) without impacting total bacterial abundance, reduced gene copy number for butyryl-CoA transferase. [42] All these results support the idea that gut microbiota-related metabolome plays a key role in CRC by providing metabolites such as butyrate.

Histopathological studies have shown that adenomatous polyps appear through a top-down morphogenesis mechanism, from the apex to the bottom of the crypts, as the more time cells reside in the mucosa, the more chances exist for epigenetic alterations through carcinogens exposure, required for tumor formation. [46] This process is in contradiction with the generally accepted statement that cancer cells derived from normal stem cells, as in the colon they exist near the base of the crypts. Therefore, in the superficial parts of the crypts, there are dysplastic epithelial cells which present markedly abnormal pattern of Ki-67 proliferation and genetic alterations of APC locus (loss of heterozygosity) leading to functional changes in $\beta$-catenin expression and localization, these mutant clones being genetically unrelated to the cells from the bottom of the crypt. [46] In the light of this particularity of the intestinal epithelium, it has been hypothesized that by influencing cell movement, profound effects on tumorigenesis may be obtained, since high-velocity cell loss represents an efficient way of eliminating cells that have acquired mutations and preventing irreversible cancerous phenotype by longer exposure to carcinogens. [47]

A study on murine $\mathrm{APC}^{+/ \mathrm{Min}}$ epithelial cells showed that in vitro, they are less motile than $\mathrm{APC}^{+++}$cells and possess a disarranged actin cytoskeletal network, properties which make them more prone to acquiring additional genetic alterations and forming tumors. [47] Treatment with two mM butyrate for 24 hours was demonstrated to increase haptotaxis in both cellular lines, acting as a promoter of the migration of colonic cancerous epithelial cells. The effect was greater in the $\mathrm{APC}^{+/ \mathrm{Min}}$ cell line, as it was able to restore both motile function and actin cytoskeletal organization seen in $\mathrm{APC}^{++}$cells. [47] The link 
between butyrate treatment and cytoskeleton assembly can be explained by its capacity of protein acetylation, which has a key role in these fibers' function. Moreover, exposure to high concentrations of butyrate $(5 \mathrm{mM})$ induced apoptosis in the mutated cells, measured by caspase-3-like activity. [47] These results may explain the protective effect determined by butyrogenic diets on CRC carcinogenesis, by increasing colonocyte velocity and shortening the exposure of cells to carcinogens, especially in the cases with APC or the $\beta$ catenin gene mutations.

Another study that assessed butyrate's effect on the motility of colonocytes investigated its ability to act directly at the molecular level of the cytoskeletal components from ileal and colonic smooth muscle cells in primary culture and on A7R5 murine cell line. [48] It was shown that butyrate $(>0.1 \mathrm{mM})$ inhibited myocytes' proliferation in A7R5 line. This finding also applied in primary culture, but only at higher concentrations, while butyrate in low concentration $(0.05-0.5 \mathrm{mM})$ significantly stimulated the proliferation of myocytes. [48] Other observed effects of butyrate included the stimulation of collagenous and noncollagenous protein synthesis, as well as enhancement of actin and myosin expression. [48] Butyrate's activity on the contractibility of colonic smooth muscle proves to be dependent on its concentration in the lumen, besides the intracellular butyrate concentration (dependent on the level of its oxidation).

The association between faecal SCFA concentrations and the efficacy of immunotherapy may emerge as a new biomarker to monitor patients undergoing treatment with programmed cell death-1 (PD-1) inhibitors. Nomura et al. recently evaluated 52 patients with solid tumors treated with nivolumab or pembrolizumab and concluded that those with higher concentrations of faecal SCFAs had a longer progression-free survival and also response to anti-PD-1 therapy. [49]

\section{Role of Omega-3 Fatty Acids in regulating butyrate-producing gut microbiota}

Consumption of omega-3 polyunsaturated fatty acids (omega-3-PUFA) rich diets has been demonstrated to be beneficial for health, supporting a good quality of life and ameliorating or preventing several disorders (cardiovascular, inflammatory, neurodegenerative diseases, diabetes mellitus and cancer). [50]

The effects of an omega-3-rich diet on gut microbiota were studied using animal models, proving there is a correlation between the two. [51] Dietary omega-3 PUFAs are largely digested in the distal intestine by anaerobic bacteria such as Bifidobacteria and Lactobacilli, influencing the intestinal flora distribution, being shown to improve gut microbial dysbiosis by increasing probiotic species and butyric acid-producing bacteria, according to several studies conducted on humans. [52]

In a published case report, a healthy 45 -year-old man who received $600 \mathrm{mg}$ of omega3 every day for 14 days had his faeces sampled. Species diversity reduced after the intervention, although butyrate-producing bacteria increased. Faecalibacterium prausnitzii and Akkermansia spp. were found to be significantly reduced. There was found a remarkable increase in Blautia, a genera whose reduction is associated with increased risk of CRC. After the 14-day washout, alterations in the gut flora were reversed, implying that the gut microbiota is a living, dynamic ecosystem that is subject to dietary changes. Therefore, increases in butyrate-producing bacteria may be responsible for some of omega-3's health advantages. [53]

Even though gut microbiota changes associated with omega-3 FUFAs are poorly understood, omega-3 fatty acids may aid in the treatment of colorectal cancer by increasing colon beneficial bacterial populations.

\section{Conclusions and future perspectives}

Given the important role of butyric acid in the prevention of CRC, therapies with exogenous SCFAs or prebiotic/probiotic administration to modulate bacterial metabolism in the gut are being proposed to reduce mucosal inflammation and induce apoptosis in cancer cells. 
Omega-3 PUFAs may affect the balance of gut microorganisms, which may contribute to the occurrence and progression of CRC, particularly due to their ability to increase butyric acid-producing bacteria.

These discoveries may shed light on the mechanisms underlying omega-3 PUFAs' impact in a variety of chronic conditions, as well as provide a framework for developing individualized medical treatments for CRC and other diseases. Supplementing the diet with omega-3 is likely to be a relevant potential mechanism for reducing CRC risk in a primary prevention setting, but it may also be relevant for the possible use of omega-3 PUFAs as adjuvant treatment of CRC.

Author Contributions: Conceptualization, D.L.S. and A.S.D.; methodology, M.P.; formal analysis, S.N.; investigation, R.I.M., I.A.K., E.A.D., L.A.K.; resources, C.V.L.; data curation, L.A.K., B.G and A.E.R.; writing-original draft preparation, A.S.D., M.P., E.A.D.; writing-review and editing, A.S.D., D.L.S., Ș.N., C.V.L.; supervision, D.L.S.; project administration, D.L.S, Ș.N. All authors have read and agreed to the published version of the manuscript.

Institutional Review Board Statement: Not applicable.

Informed Consent Statement: Not applicable.

Data Availability Statement: All data are available within the article.

Conflicts of Interest: The author Madalina Preda is an employee of MDPI; however, she does not work for the journal Nutrients at the time of submission and publication.

\section{References}

1. Vander Heiden, M.G., Cantley, L.C., \& Thompson, C.B., Understanding the Warburg effect: The metabolic requirements of cell proliferation, Science, 324(5930), 2009, 1029-1033.

2. Sung H, Ferlay J, Siegel RL. Global Cancer Statistics 2020: GLOBOCAN estimates of incidence and mortality worldwide for 36 cancers in 185 countries. CA: A Cancer Journal for Clinicians. 2021;0:1-41.

3. Rawla P, Sunkara T, Barsouk A. Epidemiology of colorectal cancer: Incidence, mortality, survival, and risk factors. Przeglad gastroenterologiczny. 2019;14(2):89, doi:10.5114/pg.2018.81072.

4. Arnold M, Sierra MS, Laversanne M, Soerjomataram I, Jemal A, Bray F. Global patterns and trends in colorectal cancer incidence and mortality. Gut. 2017;66(4):683-91, doi:10.1136/gutjnl-2015-310912.

5. Colussi D, Brandi G, Bazzoli F, Ricciardiello L. Molecular pathways involved in colorectal cancer: Implications for disease behavior and prevention. International journal of molecular sciences. 2013;14(8):16365-85, doi:10.3390/ijms140816365.

6. O'Keefe, S.J., Diet, microorganisms and their metabolites, and colon cancer, Nature Reviews Gastroenterology E Hepatology, 13(12), 2016, 691-706.

7. Francescone, R., Hou, V., Grivennikov, S.I., Microbiome, inflammation and cancer, Cancer Journal, 20(3), $2014,181$.

8. Saus, E., Iraola-Guzmán, S., Willis, J.R., Brunet-Vega, A., Gabaldón, T., Microbiome and colorectal cancer: Roles in carcinogenesis and clinical potential, Molecular aspects of medicine, 69, 2019, 93-106.

9. Scott AJ, Alexander JL, Merrifield CA; et al. International Cancer Microbiome Consortium consensus statement on the role of the human microbiome in carcinogenesis. Gut. 2019;68(9):1624-32, doi:10.1136/gutjnl-2019-318556.

10. Hornef M. Pathogens, commensal symbionts, and pathobionts: Discovery and functional effects on the host. ILAR journal. 2015;56(2):159-62, doi:10.1093/ilar/ilv007.

11. Rojo D, Méndez-García C, Raczkowska BA; et al. Exploring the human microbiome from multiple perspectives: Factors altering its composition and function. FEMS microbiology reviews. 2017;41(4):453-78, doi:10.1093/femsre/fuw046.

12. Blaser MJ. The microbiome revolution. The Journal of clinical investigation. 2014;124(10):4162-5, doi:10.1172/JCI78366.

13. Tibbs TN, Lopez LR, Arthur JC. The influence of the microbiota on immune development, chronic inflammation, and cancer in the context of aging. Microbial Cell. 2019;6(8):324, doi:10.15698/mic2019.08.685.

14. Sommer, F., Anderson, J.M., Bharti, R., Raes, J., \& Rosenstiel, P., The resilience of the intestinal microbiota influences health and disease, Nature Reviews Microbiology, 15(10), 2017, 630-638.

15. Noecker C, McNally CP, Eng A, Borenstein E. High-resolution characterization of the human microbiome. Translational Research. 2017;179:7-23, doi:10.1016/j.trsl.2016.07.012.

16. Lagier JC, Dubourg G, Million M, Cadoret F, Bilen M, Fenollar F, Levasseur A, Rolain JM, Fournier PE, Raoult D. Culturing the human microbiota and culturomics. Nature Reviews Microbiology. 2018;16(9):540-50, doi:10.1038/s41579-018-0041-0.

17. Caputo A, Fournier PE, Raoult D. Genome and pan-genome analysis to classify emerging bacteria. Biology direct. 2019;14(1):19. https://doi.org/10.1186/s13062-019-0234-0.

18. Mchardy, I.H., Goudarzi, M., Tong, M., Ruegger, P.M., Schwager, E., Weger, J.R., Mcgovern, D.P., Integrative analysis of the microbiome and metabolome of the human intestinal mucosal surface reveals exquisite inter-relationships, Microbiome, 1(1), 2013, 17. 
19. Chittim, C.L., Irwin, S.M., \& Balskus, E.P., Deciphering Human Gut Microbiota-Nutrient Interactions: A Role for Biochemistry, Biochemistry, 57(18), 2018, 2567-2577.

20. Kovács, T., Mikó, E., Ujlaki, G., Sári, Z., Bai, P., The Microbiome as a Component of the Tumor Microenvironment, In Tumor Microenvironment, Springer, Cham, 2020, 137-153.

21. Weir, T.L., Manter, D.K., Sheflin, A.M., Barnett, B.A., Heuberger, A.L., Ryan, E.P., Stool microbiome and metabolome differences between colorectal cancer patients and healthy adults. PLoS ONE, 8(8), 2013, e70803.

22. Sheflin, A.M., Whitney, A.K., Weir, T.L., Cancer-promoting effects of microbial dysbiosis, Current oncology reports, 16(10), 2014, 406.

23. Baffy, G., Gut Microbiota and Cancer of the Host: Colliding Interests, Advances in experimental medicine and biology, 1219, 2020, 93-107.

24. Malcomson, F.C., Breininger, S.P., Elgendy, K., Joel, A., Ranathunga, R.M.T.K., Hill, T.R., Mathers, J. C, Design and baseline characteristics of the Biomarkers Of Risk In Colorectal Cancer (BORICC) Follow-Up study: A 12+ years follow-up, Nutrition and health, 25(3), 2019, 231-238.

25. Canani, R.B., Di Costanzo, M., Leone, L., Pedata, M., Meli, R., Calignano, A., Potential beneficial effects of butyrate in intestinal and extraintestinal diseases, World journal of gastroenterology, 17(12), 2011, 1519-1528.

26. Flint, H.J., Duncan, S.H., Scott, K.P., Louis, P., Links between diet, gut microbiota composition and gut metabolism. Proceedings of the Nutrition Society, 74(1), 2015, 13-22.

27. Pattayil L, Balakrishnan-Saraswathi HT. In vitro evaluation of apoptotic induction of butyric acid derivatives in colorectal carcinoma cells. Anticancer research. 2019;39(7):3795-801.

28. Campos-Perez W, Martinez-Lopez E. Effects of short chain fatty acids on metabolic and inflammatory processes in human health. Biochimica et Biophysica Acta (BBA)-Molecular and Cell Biology of Lipids. 2021:158900.

29. Hajjar R, Richard CS, Santos MM. The role of butyrate in surgical and oncological outcomes in colorectal cancer. American Journal of Physiology-Gastrointestinal and Liver Physiology. 2021.

30. Louis, P., \& Flint, H.J., Diversity, metabolism and microbial ecology of butyrate-producing bacteria from the human large intestine, FEMS microbiology letters, 294(1), 2009, 1-8.

31. Hibberd, A.A., Lyra, A., Ouwehand, A.C., Rolny, P., Lindgren, H., Cedgård, L., \& Wettergren, Y, Intestinal microbiota is altered in patients with colon cancer and modified by probiotic intervention. BMJ open gastroenterology, 4(1), 2017 e000145.

32. Wang, T., Cai, G., Qiu, Y., Fei, N., Zhang, M., Pang, X., Zhao, L., Structural segregation of gut microbiota between colorectal cancer patients and healthy volunteers, The ISME Journal, 6(2), 2012, 320-329.

33. Chen, W., Liu, F., Ling, Z., Tong, X., \& Xiang, C., Human intestinal lumen and mucosa-associated microbiota in patients with colorectal cancer. PLoS ONE, 7(6), 2012, e39743.

34. Hippe, B., Zwielehner, J., Liszt, K., Lassl, C., Unger, F., Haslberger, A.G., Quantification of butyryl CoA: Acetate CoA-transferase genes reveals different butyrate production capacity in individuals according to diet and age, FEMS microbiology letters, 316(2), 2011, 130-135.

35. Feinberg, A.P., The key role of epigenetics in human disease prevention and mitigation, New England Journal of Medicine, 378(14), 2018, 1323-1334.

36. Turner, B.M., Histone acetylation and an epigenetic code, Bioessays, 22(9), 2000, 836-845.

37. Riggs, M.G., Whittaker, R.G., Neumann, J.R., \& Ingram, V.M., n-Butyrate causes histone modification in HeLa and Friend erythroleukaemia cells, Nature, 268(5619), 1977, 462-464.

38. Zagni, C., Floresta, G., Monciino, G., \& Rescifina, A., The search for potent, small-molecule HDACIs in cancer treatment: A decade after vorinostat, Medicinal research reviews, 37(6), 2017, 1373-1428.

39. Cao K, Wang G, Li W, Zhang L, Wang R, Huang Y, Du L, Jiang J, Wu C, He X, Roberts AI. Histone deacetylase inhibitors prevent activation-induced cell death and promote anti-tumor immunity. Oncogene. 2015;34(49):5960-70.

40. Archer SY, Johnson J, Kim HJ, Ma Q, Mou H, Daesety V, Meng S, Hodin RA. The histone deacetylase inhibitor butyrate downregulates cyclin B1 gene expression via a p21/WAF-1-dependent mechanism in human colon cancer cells. American Journal of Physiology-Gastrointestinal and Liver Physiology. 2005;289(4):G696-703.

41. Bachem A, Makhlouf C, Binger KJ; et al. Microbiota-derived short-chain fatty acids promote the memory potential of antigenactivated CD8+ T cells. Immunity. 2019;51(2):285-97.

42. Belcheva, A., Irrazabal, T., Robertson, S.J., Streutker, C., Maughan, H., Rubino, S., Green, B., Gut microbial metabolism drives transformation of MSH2-deficient colon epithelial cells, Cell, 158(2), 2014, 288-299.

43. Aceto, G.M., Catalano, T., \& Curia, M.C., Molecular Aspects of Colorectal Adenomas: The Interplay among Microenvironment, Oxidative Stress, and Predisposition, BioMed Research International 2020, 2020, 1726309.

44. Gnagnarella, P., Gandini, S., La Vecchia, C., \& Maisonneuve, P., Glycemic index, glycemic load, and cancer risk: A meta-analysis, The American journal of clinical nutrition, 87(6), 2008, 1793-1801.

45. Meyerhardt, J.A., Sato, K., Niedzwiecki, D., Ye, C., Saltz, L.B., Mayer, R.J., Wigler, D.S., Dietary glycemic load and cancer recurrence and survival in patients with stage III colon cancer: Findings from CALGB 89803, Journal of the National Cancer Institute, 104(22), 2012, 1702-1711.

46. Shih, I.M., Wang, T.L., Traverso, G., Romans, K., Hamilton, S.R., Ben-Sasson, S., ... \& Vogelstein, B., Top-down morphogenesis of colorectal tumours, Proceedings of the National Academy of Sciences, 98(5), 2001, 2640-2645.

47. Forest, V., Clement, M., Pierre, F., Meflah, K., \& Menanteau, J., Butyrate restores motile function and actin cytoskeletal network integrity in APC mutated mouse colon epithelial cells, Nutrition and cancer, 45(1), 2003, 84-92. 
48. Le Blay, G., Blottiere, H.M., Ferrier, L., Le Foll, E., Bonnet, C., Galmiche, J.P., \& Cherbut, C., Short-chain fatty acids induce cytoskeletal and extracellular protein modifications associated with modulation of proliferation on primary culture of rat intestinal smooth muscle cells, Digestive diseases and sciences, 45(8), 2000, 1623-1630.

49. Nomura M, Nagatomo R, Doi K; et al. Association of short-chain fatty acids in the gut microbiome with clinical response to treatment with nivolumab or pembrolizumab in patients with solid cancer tumors. JAMA network open. 2020;3(4):e202895.

50. Weylandt KH, Serini S, Chen YQ, Su HM, Lim K, Cittadini A, Calviello G. Omega-3 polyunsaturated fatty acids: the way forward in times of mixed evidence. BioMed research international. 2015 Aug 2;2015.

51. Druart C, Neyrinck AM, Vlaeminck B, Fievez V, Cani PD, Delzenne NM. Role of the lower and upper intestine in the production and absorption of gut microbiota-derived PUFA metabolites. PloS one. 2014 Jan 27;9(1):e87560.

52. Watson H, Mitra S, Croden FC, et al. A randomised trial of the effect of omega-3 polyunsaturated fatty acid supplements on the human intestinal microbiota. Gut. 2018 Nov 1;67(11):1974-83.

53. Noriega BS, Sanchez-Gonzalez MA, Salyakina D, Coffman J. Understanding the impact of omega-3 rich diet on the gut microbiota. Case reports in medicine. 2016 Mar 14;2016. 